\title{
A Hierarchical Secure Ring-Oriented Multicast Protocol over Mobile Ad Hoc Network ${ }^{\star}$
}

\author{
Choong Seon Hong and Yubai Yang \\ Department of Computer Science, Kyung Hee University, \\ 1 Seocheon, Giheung, Yongin, Gyeonggi 449-701 Korea \\ cshong.khu.ac.kr, yyb@networking.khu.ac.kr
}

\begin{abstract}
In this paper, we propose a novel scheme of Hierarchical Eulerian Ring-Oriented Multicast Protocol over mobile ad hoc network. It has features that concentrate on efficiency and robustness simultaneously. It is also an application-driven proposal for hazard detection. Simulation results show different level of improvements on control traffic, end-to-end delay by comparing with tree-based and mesh-based multicast protocols.
\end{abstract}

Keywords: Hierarchical, Eulerian Ring, Multicast, Ad hoc network.

\section{Proposed Scheme}

We employ Fisheye State Routing (FSR) protocol as our IP protocol, with which we can get knowledge within predefined-scope. FSR introduces the notion of multi-layer fisheye scope to reduce routing update overhead in large-scale networks. Mobile nodes exchange link state entries with their neighbors with a frequency that depends on distance to destination. With the help of FSR as IP protocol, our proposal can get the necessary information within predefined scope that is the basis of searching algorithm for hierarchical Eulerian ring.

\subsection{A Novel Eulerian Ring Based Scheme}

Let $\mathrm{G}$ be a planar graph, stands for a group of mobile nodes in ad hoc network. Nodes in $\mathrm{G}$ are denoted by $\mathrm{V}(\mathrm{G})$; links between nodes in $\mathrm{G}$ are denoted by $\mathrm{E}(\mathrm{G})$ (we discuss only bi-direction link herein); The degree of $\mathrm{V}$ is the number of edges meeting at $\mathrm{V}$, and is denoted by deg $\mathrm{v}$. If there is a closed trail that includes every edge once and only, such a trail is called a Eulerian trail. If it forms a ring, it is called a Eulerian ring. Proofs of theorems are omitted, details could be found in [2]. The reason that we employ Eulerian Ring is edge-traceable. In Eulerian graph we could walk along every link once and only once. First we make the following definitions for terminology and rule: Theorem 1: let $\mathrm{G}$ be a connected

\footnotetext{
* This work was supported by University ITRC Project of MIC, Dr. C.S.Hong is the corresponding author.
} 
graph, then $G$ is Eulerian if and only if every vertex of $G$ has even degree. Center node: who provides FSR link-state information of the region. Multicast receiver: node who receives multicast packets. $E_{i, j}$ : a link connects node i and node j. Leaf node: a node whose deg v=1. Even node: node with even degree. Odd node: node with odd links. Rule 1: let neighbor take leaf node's task (note that leaf node has only one neighbor), then delete leaf nodes. Execute repeatedly until there is no leaf node in G. Calculate degree then mark node type (even node and odd node). 2-odd-link: link that connects 2 odd nodes. 2-even-link: link that connects 2 even nodes. Odd-even-link: link that connects one odd node and one even node. Rule 2: mark link type (2-odd-link, 2-even-link or odd-even-link). Maintain 2-evenlink unchanged, prune 2-odd-link in descending degree (from maximum degree to minimum degree), and is denoted by freeze-link. Execute rule 2 repeatedly until no 2-odd-link exists. Theorem 2: In a graph $\mathrm{G}$, the number of nodes of odd degree is even. After rule 2, only odd-even-links remain. And odd nodes will appear in pairs Rule 3: Mark odd node pairs in descending degree. Find path connected them in descending way, mark it recursive-freeze-link and prune it. If new odd node generates, execute rule 3 recursively until no odd-node-pair exists. Main ring: ring contains center node. Assist ring: ring other than main ring but connects to main ring. It receives multi-cast packets from diverge nodes. Branch: a tree or line connects to ring. It contains at least one multicast receiver and have a joint to a ring. Master: a node that takes charge in multicast service for his ring or branch. Each ring, assist ring and branch should have its own master respectively. The master of assist ring is also referred as sub-master. Member: node that takes part in multicast affairs, including multicast member and forwarding node. Diverge node: a node resides on joint of rings or joint of ring and branch. It duplicates multicast packet for the lower ring or branch. Bridge: the only link without which graph will be disconnected. $\delta$ : A time-out setting to prevent situations in which a node has waited too long for a token. For example, due to crash failure of node or link, a message taking time unites longer than for transmission is considered as having been lost. Theorem 3: if $\mathrm{G}$ is a graph in which every vertex has even degree, then $\mathrm{G}$ can be spitted into disjoint cycles-that is, no two cycles have any edges in common. Rule 4: execute Fleury algorithm [2], then split it into small circles according to theorem 3; construct ring-based topology by marking main ring, assist ring and branch. Recover the freeze-link and recursive-freeze-link, make them part of branch or assist ring if it contains member, and so does the leaf node generated in Rule 1.

\subsection{Hierarchy}

The second character in the protocol is hierarchy, which is achieved by topology of different level Eulerian rings and branches. The hierarchical architecture has 2 advantages, multicast routing updates and control traffic could be restricted locally to reduce delivery overhead; On the other hand it may shorten subscription time by registering to a nearer multicast receiver rather than a remote multicast source. As shown in Fig.1, the main ring p, q, r, e, d, o is the first level; branch $\mathrm{o}, \mathrm{c}, \mathrm{x}$ and assist ring $\mathrm{p}, \mathrm{q}, \mathrm{r}, \mathrm{f}, \mathrm{h}, \mathrm{i}, \mathrm{k}, \mathrm{l}$ are the second level. To achieve hier- 


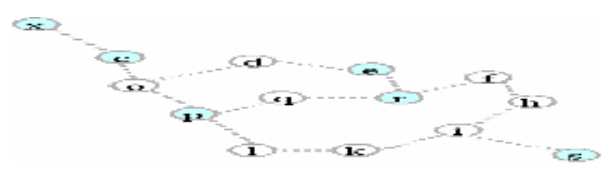

Fig. 1. Hierarchical Eulerian ring-oriented topology

archy we employ master and diverge node. Each ring and branch would have a master. Master of main ring is honored to manage its members including diverge nodes; the diverge node stores information of assist ring or branch in lower level; assist ring master play the same role in their own rings as main ring master. Both master and diverge node are the basis of hierarchical architecture. Note, each node belongs to one but only master from whom it gets multicast packets. Diverge node locating at the joint point has dual duty, one is to do the work of his own ring such as forwarding multicast packets, relaying token in appropriate direction and reporting error message; the other is to establish hierarchical level by transferring multicast packets to lower level.

\subsection{Master Election}

The algorithm of electing master should be carefully considered because it is the master's duty to perform multicast task and ring recovery. In order to simplify our model, we suppose each mobile node has same computation capability and choose only 2 parameters for master election: average error packets rate and battery capacity. The former is a kind of BER, and proposed by [3]; the latter emphasizes the lifetime of power, and it is proposed by [4]. $N e w_{P_{k}^{e}[i, j]}$ is defined as average error packet within sampling period $\mathrm{K}$ for a given direction-oriented wireless link from i to $\mathrm{j}, P_{k}^{e}[i, j]$ as average error packet after sampling period $\mathrm{K}$ for a given direction-oriented wireless link from $i$ to $j$.

$$
P e_{[\mathrm{i}, \mathrm{j}]}^{k}=\left(\mu \times P e_{[i, j]}^{k-1}\right)+\left((1-\mu) \times N e w_{-} P e_{[i, j]}^{k}\right) \quad i, j V, 1>u>0
$$

$L T_{i}^{k}$ is defined as live time estimated after sampling period. $L T_{i}^{k}$ is calculated as:

$$
L T_{\mathrm{i}}^{k}=\rho_{i}^{-1} \times\left(F_{i} / E_{i(t)}\right)^{-\alpha}
$$

Herein $E_{i(t)}$ means remaining battery capacity of node i at time t; $F_{i}$ is fullcharge battery capacity of node $\mathrm{i} ; \alpha$ is a positive weighting factor for ratio of the remaining; $\rho_{i}$ stands for the transmit power at node $\mathrm{i}$ at time $\mathrm{k}$. We define $\mathrm{Pi}$ as the possibility that a node has to become a master:

$$
P_{\mathrm{i}}=\left(L T_{i}^{k}\right) \times\left(\sum P e_{[i, j]}^{k} / N\right)
$$

The $\sum \mathrm{P}_{\mathrm{e}[\mathrm{i}, \mathrm{j}]}^{\mathrm{k}} / \mathrm{N}$ means the average error rate among links processed by node $\mathrm{i}$, and $L T_{i}^{k}$ indicates the live time that $\mathrm{Ni}$ maybe last after sampling period, the longer live time means the higher possibility to be elected as master. 


\section{Simulation Result and Conclusion}

Mobile hosts are placed randomly within a $1000 \mathrm{~m} * 1000 \mathrm{~m}$ area and moved randomly. Radio propagation range is $250 \mathrm{~m}$. The number of mobile host is 40 and member of multicast group increases from 0 to 30 , ranging in the set $0,5,10,15$, $20,25,30$. The channel capacity is $2 \mathrm{Mbits} / \mathrm{s}$ and buffer size is $64000 \mathrm{bits}$ The $\mathrm{X}$-axis in Fig.2 stands for the number of multicast member (MM). The Y-axis stands for end-to-end delay in average and its unit is ms. End-to-end delay of our proposal increases smoothly, ranging from $1 \mathrm{~ms}$ to $2 \mathrm{~ms}$ as the number of multicast member increases. In this paper, a novel model of Hierarchical Eulerian Ring-Oriented Multicast Protocol is proposed. A graph based multicast schemes including hierarchical architecture, multicast agent and relative algorithms have been discussed. Simulation results indicate that the proposed scheme outperforms ARMIS, CAMP and ITAMAR in terms of service latency to some extent. We hope our scheme could shed a little light of graphoriented multicast scheme over mobile ad hoc network.

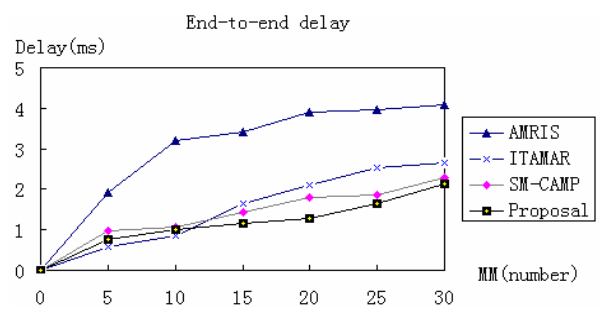

Fig. 2. End-to-end delay

\section{References}

1. Carlos de Morais Cordeiro, H. Gossain, and D.P. Agrawal, "Multicast over Wireless Mobile Ad Hoc Networks: Present and Future Directions, " IEEE Network, Vol.17, No.1, Jan.2003.

2. R.J. Wilson and J.J.Watkins, Graphs: an introductory approach, John Wiley and Sons, Inc Page 122-127.

3. Ying chun, liu yong, shi meilin, "A hybrid protocol in Ad hoc network", 2001 journal of software, China.

4. M. Maleki, K. Dantu and M. Pedram, "Power-aware Source Routing Protocol for Mobile Ad hoc Network", International Symposium on Low Power Electronics and Design, 2002 IEEE. 